\title{
Histopathology of the gut in rheumatic diseases
}

\author{
F. Macaluso', G. Guggino', A. Rizzo ${ }^{2}$, A. Ferrante ${ }^{1}$, F. Ciccia ${ }^{1}$ \\ ${ }^{1}$ Rheumatology Unit, University of Palermo; \\ ${ }^{2}$ Pathology Unit, Azienda Ospedaliera Villa Sofia Cervello, Palermo, Italy
}

\section{SUMMARY}

The gastrointestinal tract regulates the trafficking of macromolecules between the environment and the host through an epithelial barrier mechanism and is an important part of the immune system controlling the equilibrium between tolerance and immunity to non-self-antigens. Various evidence indicates that intestinal inflammation occurs in patients with rheumatic diseases. In many rheumatic diseases intestinal inflammation appears to be linked to dysbiosis and possibly represents the common denominator in the pathogenesis of different rheumatic diseases. The continuative interaction between dysbiosis and the intestinal immune system may lead to the aberrant activation of immune cells that can re-circulate from the gut to the sites of extraintestinal inflammation as observed in patients with ankylosing spondylitis. The exact contribution of genetic factors in the development of intestinal inflammation in rheumatic diseases needs to be clarified.

Key words: Intestinal inflammation; Symbiosis; Ankylosing spondylitis; Rheumatoid arthritis; Behçet's disease; Systemic lupus erythematosus; Systemic sclerosis.

Reumatismo, 2018; 70 (3): 178-186

\section{INTRODUCTION}

The gastrointestinal (GI) tract is an organ system within humans that digests food to extract and absorb energy and nutrients, expelling the remaining waste as faeces and urine. In particular, the GI tract regulates the trafficking of macromolecules between the environment and the host through an epithelial barrier mechanism (1). The Gl tract is an important part of the immune system: the intestinal epithelial barrier, together with the gut-associated lymphoid tissue and the neuroendocrine network, play in fact a fundamental role in controlling the equilibrium between tolerance and immunity to nonself-antigens (1). Dysregulation of this organ, in genetically susceptible individuals, may lead to both intestinal and extra-intestinal autoimmune disorders (1). The large intestine hosts many trillions of microbes, the so-called microbiota, that can deal with molecules that the human body cannot otherwise break down in a perfect example of symbiosis (2). These microorganisms play metabolic, trophic and protective roles and participate in normal human development and homoeostasis, also preventing the overgrowth of potentially harmful bacteria (2). It is intuitive to imagine that a continuous interchange occurs between the host organism and the microbiota. The perturbation of this dialogue results in the so-called dysbiosis and there is emerging literature on its role in the pathogenesis of systemic autoimmune disease such as type 1 diabetes, celiac disease, ankylosing spondylitis and rheumatoid arthritis (3-6). This review aims to summarise current data on the role of the gastrointestinal involvement in the pathogenesis of systemic rheumatic disease, focusing on GI histopathological changes.

\section{HISTOLOGICAL CHANGES IN THE GUT OF SPONDYLOARTHRITIS PATIENTS}

Spondyloarthropathy ( $\mathrm{SpA}$ ) refers to a group of articular inflammatory diseases that share common genetic, clinical and radiological features in addition to their 
association with the human leukocyte antigen (HLA)-B27 antigen (7-9). The hallmark of the disease is the inflammation of the bone marrow located in the spine and in extra-spinal sites (9). Although the pathogenesis of $\mathrm{SpA}$ is still elusive, activation of innate and adaptive immune responses has been reported in SpA patients (7). The prototype of these diseases is ankylosing spondylitis (AS). Other diseases include reactive arthritis, psoriatic arthritis (PsA) and arthritis associated with inflammatory bowel disease (10). The role of the gastrointestinal tract in the pathogenesis of $\mathrm{SpA}$ is strongly suggested by a growing body of evidence. In particular, a subclinical gut inflammation documented by ileocolonoscopy (macroscopical or microscopical lesions) is present in up to $60 \%$ of patients with $\mathrm{SpA}$, asymptomatic for signs and symptoms of intestinal inflammation (11-14). SpA-associated gut inflammation seems to be not only an epiphenomenon of the ongoing systemic inflammatory process. It has in fact been demonstrated that gut inflammation is an important prognostic factor in $\mathrm{SpA}$, being associated with more extensive bone marrow edema of the sacroiliac joints and a higher risk of progression to ankylosing spondylitis (AS) and of developing CD (13).

\section{ANKYLOSING SPONDYLITIS}

Historically, the histological appearance of intestinal lesions in ankylosing spondylitis (AS) patients has been referred to as the acute or chronic type of inflammation (12). The acute type resembles a self-limiting bacterial enterocolitis characterized by the preservation of mucosal architecture, infiltration of ileal villi and crypt epithelium with polymorphonuclear cells and an increased number of inflammatory cells in the lamina propria $(12,15)$. The chronic type of inflammation resembles chronic ileocolitis and is characterized by the distortion of crypts, atrophy of the villous surface of the colonic mucosa, blunting and fusion of villi, increased mixed lamina propria cellularity and basal lymphoid aggregates in the propria inflammation of- ten resembling the ileocolitis observed in Crohn's disease $(12,15)$. In some cases of chronic lesions, aphthoid ulcers, branching of the crypts, pseudo-pyloric metaplasia and Crohn's disease-like granulomas are present. It is unclear whether the acute and chronic forms are a continuation of the same histopathological spectrum and whether there is a transition from one form to another, and in which percentage of patients one can observe a histological healing. What we do know is that up to $10 \%$ of patients with chronic gut inflammation will develop over the time a clinically overt CD (14). More recently, however, several studies from our group have demonstrated the occurrence of specific histological changes in AS gut independently of the degree of intestinal inflammation. Goblet cells hyperplasia and increased mucin production has in fact been proved in AS and correlated with the increased expression of IL-22, a protective cytokine (16). Paneth cells (PC), along with goblet cells, enterocytes, and enteroendocrine cells, represent the principal cell types of the epithelium of the small intestine (17). They are localized below the intestinal stem cells in the intestinal glands (crypts of Lieberkühn) and are characterized by the presence of large eosinophilic refractile granules in their cytoplasm. PC secrete antimicrobial peptides in response to bacterial antigens into the lumen of the intestinal gland, thereby contributing to maintenance of the gastrointestinal barrier (17). Activation of PC, characterized by the increased production of antimicrobial peptides, has been demonstrated in AS gut (17). In addition, PC represents the most important site where the production of IL-23, a key cytokine involved in the regulation of innate and adaptive immune responses, takes place in AS (15). Other histological alterations are represented by the detachment of epithelial cells from the basal membrane and the occurrence of vasculitic lesions, mainly represented by intense hemorrhagic extravasation in the context of the lamina propria. Interestingly, similar tissue damages have been described as a consequence of the response of epithelial cells to bacte- 
rial toxins (1). In a recent paper, the presence of these histological alterations has been correlated in AS with the presence of adherent and invading bacteria (3). The presence of intestinal dysbiosis, confirmed by other studies, might be responsible for the alteration of the intestinal epithelial and vascular barrier observed in AS gut (18). In healthy individuals, the intestinal microbiota cannot access the liver, spleen, or other peripheral tissues. Some pathogenic bacteria can reach these sites, however, and can induce a systemic immune response. Such compartmentalization is guaranteed by the presence of an epithelial gut barrier (consisting of a single-cell layer) and of a gut-vascular barrier (GVB) that controls the translocation of antigens into the blood stream and prohibits entry of the microbiota $(1,19)$. Disruption of the intestinal gut barrier and GVB occurs in AS patients and is linked to the presence of bacteria that induce the production of zonulin (20). Zonulin (haptoglobin 2 precursor) is a protein that modulates the permeability of tight junctions between cells of the wall of the digestive tract. In AS gut, Zonulin expression is up-regulated and modulated by isolated intestinal bacteria (18). The functional relevance of zonulin up-regulation seems to be linked to the disruption of the epithelial and vascular intestinal barrier (18). The relative contribution of the genetic factors involved in SpA pathogenesis in influencing these histological changes cannot be excluded and requires further study. Given the absence of reliable markers, the diagnosis of intestinal inflammation in $\mathrm{SpA}$ is essentially made by means of endoscopy. Recently, however, elevated serum calprotectin and c-reactive protein (CRP) have been demonstrated to be independently associated with microscopic bowel inflammation in AS (21). In the presence of intestinal inflammation, fecal calprotectin is elevated, indicating the migration of neutrophils to the inflamed intestinal mucosa. In this study, the Authors suggest a screening approach where initially serum calprotectin and CRP are assessed and, if necessary, fecal calprotectin. The model using this scenario provided an area under the ROC curve of $74.4 \%$ for detection of bowel inflammation (21).

\section{PSORIATIC ARTHRITIS}

Gut inflammation has been demonstrated to occur also in patients affected by psoriatic arthritis (PsA) (22). Schatteman et al demonstrated that gut inflammation is only present in PsA subgroups that belong to the spondyloarthropathy concept and is associated with HLA-B27 and Bw62 (22). In their study, the prevalence of gut inflammation in psoriatic $\mathrm{SpA}$ was significantly lower than in non-psoriatic SpA. In a subsequent study, Mielants and co-workers (12) prospectively evaluated the clinical evolution of different forms of $\mathrm{SpA}$ in relation to the type of gut histology in ileocolonoscopic biopsy specimens. Gut inflammation, mainly subclinical, could be demonstrated in $68 \%$ of patients with $\mathrm{SpA}$ (10) and patients with non-AS-SpA and with inflammatory gut lesions have greater risk of developing AS. On the other hand, the presence of chronic gut inflammation, the persistence of high inflammatory serum variables and HLA-B27 negativity in the presence of sacroiliitis or AS are risk factors for developing inflammatory bowel diseases (23). Scarpa et al evaluated colonic mucosa of patients with both active psoriasis and psoriatic arthritis (PsA) without bowel symptoms (24). In their study, $40 \%$ of the patients enrolled had macroscopically normal colonic mucosa. In the remaining $60 \%$ reddening was frequently recorded, while edema and granular changes appeared less commonly. Friability was markedly rare and bleeding and ulcerations were absent. All the patients showed microscopic changes mainly characterized by the increase in lamina propria cellularity (consisting of plasma cells and lymphocytes) and lymphoid aggregates were found in all cases (24). Active inflammation, evident as neutrophilic polymorphonuclear cell infiltration also frequently occurred. Glandular atrophy, mucosal surface changes and crypt abnormalities were rarely observed. The occurrence of subclinical 
gut inflammation in PsA has been recently confirmed by our group in a recent study (25). In this study, PsA subclinical gut inflammation was characterized by a clear Th17 and Th22, but not Th1, polarized immune response. In contrast with AS and $\mathrm{CD}$, a strong and significant up-regulation of IL-9 was observed in PsA gut, especially among infiltrating mononuclear cells, high endothelial venules, and PC (25). IL-9-positive mononuclear cells were demonstrated to be in large part Th9 cells and IL-9 overexpression was accompanied by significant PC hyperplasia. PC strongly overexpressed IL-9R, and stimulation of epithelial cells, isolated from PsA patients, with IL-9 resulted in overexpression of $\alpha$-defensin 5 and IL-23p19, indicating a role for IL-9 in modulating PC function (25). Similarly to AS, subclinical gut inflammation in PsA patients appears to be accompanied by the presence of a specific microbiological signature (26). In this regard, Scher et al recently demonstrated that the gut microbiota observed in patients with PsA and patients with skin psoriasis is less diverse when compared to that in healthy controls (26). This could be attributed to the reduced presence of several taxa. Samples from both patient groups showed a relative decrease in abundance of Coprococcus species, while samples from PsA patients were also characterized by a significant reduction in Akkermansia, Ruminococcus, and Pseudobutyrivibrio. Supernatants of fecal samples from PsA patients revealed an increase in sIgA levels and decrease in RANKL levels. Analysis of fatty acids revealed low fecal quantities of hexanoate and heptanoate both in patients with PsA and patients with psoriasis.

\section{JUVENILE CHRONIC ARTHRITIS}

Several studies have demonstrated the occurrence of histological changes in patients with juvenile chronic arthritis. Mielants et al. (27) performed ileocolonoscopy with biopsy of the ileum, ileocecal valve and cecum on 32 patients presenting juvenile chronic arthritis (JIA) with pauci-articular onset. Twenty-two patients (68\%) were HLA-B27 positive and 8 patients $(25 \%)$ carried the HLA-Bw62 antigen. In this study, in the majority of patients presenting JIA with pauci-articular onset, gut inflammation was found on ileum biopsy. In particular, fourteen patients had macroscopic lesions of the ileum and ileocecal valve although only 8 of these had a history of intestinal complaints (27). Twenty-six patients $(81 \%)$ presented histological signs of gut inflammation mostly classified as active chronic or Crohn-like lesions. Only 7 patients had acute or bacterial enteritislike lesions. All the HLA-Bw62 positive patients had histological evidence of gut inflammation with chronic or Crohn-like lesions. The presence of chronic gut inflammation on gut biopsy seems to be a predictive factor in juveniles for evolution to AS (28). In a subsequent study, Pichler et al evaluated the histopathological features of gastrointestinal mucosal biopsies in children with juvenile idiopathic arthritis (29). Of the 33 children enrolled, 28 (85\%) had gut mucosal inflammation, mostly affecting the colon (80\%). Active inflammation of the gut was found in 5 out of 28 (17\%) children, while 15 out of $28(53 \%)$ children showed mild nonspecific inflammation. Eight patients (27\%) had predominantly an eosinophilic infiltrate (29). The Authors demonstrated a negative association with the use of immunosuppressors and the presence of eosinophil inflammation (29). Gut microflora and dysbiosis have been also linked to the pathogenesis of juvenile idiopathic arthritis. Di Paola et $\mathrm{al}$ in a recent study compared the faecal microbiota composition of JIA patients with healthy subjects (HS), evaluating differences in microbial profiles between subcategories of JIA, such as enthesitis-related arthritis (JIA-ERA), in which inflammation of entheses occurs, and polyarticular JIA, non-enthesitis related arthritis (JIA-nERA) (30). Through taxon-level analysis, the authors discovered alteration of faecal microbiota components. In particular, abundance in Ruminococcaceae in both JIA categories, reduction in Clostridiaceae and Peptostreptococcaceae in JIA-ERA, and increase 
in Veillonellaceae in JIA-nERA were demonstrated respectively, compared with controls (30). Among the more relevant genera, the authors found an increase in Clostridium cluster XIVb, involved in colitis and arthritis, in JIA-ERA patients compared with controls, and a trend of decrease in Faecalibacterium, known for anti-inflammatory properties, in JIA-nERA compared with JIA-ERA and controls. Differential abundant taxa identified JIA patients for the HLA-B27 allele, including Bilophila, Clostridium cluster XIVb, Oscillibacter, and Parvimonas. Prediction analysis of metabolic functions showed that JIA-ERA metagenome was differentially enriched in bacterial functions related to cell motility and chemotaxis, suggesting selection of potential virulence traits (30). Finally, the authors demonstrated differential microbial profiles and intra-group variability among active disease and remission, suggesting instability of the microbial ecosystem in autoimmune diseases (30). Gut microbiome in children with enthesitis-related arthritis in a developing country and the effect of probiotic administration have been recently studied (31). In this study, patients showed abundance of Bacteroidaceae and Enterobacteriaceae families and a decreased representation of Prevotellaceae compared to controls. Also, genera Bacteroides, Enterococcus and Klebsiella were over-represented and genus Prevotella was underrepresented in ERA patients. Interestingly, Probiotic therapy led to a non-significant increase in Prevotellaceae (31).

\section{HISTOLOGIC CHANGES IN THE GUT OF BEHCET'S DISEASE PATIENTS}

Behçet's disease is a rare, chronic, autoimmune, auto-inflammatory disorder of unknown origin that results in inflammation of the blood vessels and tissues. Behçet's disease involves the gastrointestinal tract in $10-15 \%$ of cases with localized lesions in the ileocecal region (32). The clinical picture is heterogeneous with various clusters of disease expression. Asakura et al. (33) demonstrated the presence of marked lym- phangiectasia in the lamina propria of biopsy specimens of jejunal mucosa and the presence of ulcers and healed ulcers of the large intestine. Electron microscopic studies showed that there were thousands of precipitated lymph protein bodies in the extracellular spaces of the lamina propria. A recent study from our group demonstrated the occurrence of a subclinical gut inflammation in patients with Behçet's disease accompanied by a predominant Th1, but not a Th17, response (34). Gut microbiota compositional alteration might be associated with immune dysfunction in patients with Behcet's disease. In particular, Shimizu et al. demonstrated that the genera Bifidobacterium and Eggerthella are increased significantly and the genera Megamonas and Prevotella decreased significantly in Behçet's disease patients compared with normal individuals (35). A linear discriminant analysis (LDA) of bacterial taxa showed that the phylum Actinobacteria, including Bifidobacterium, and the family Lactobacillaceae exhibited larger positive effect sizes than other bacteria in patients with Behçet's disease. Consolandi et al. recently found that Behçet's patients were significantly depleted in the genera Roseburia and Subdoligranulum and showed a significant decrease of butyrate production. Since that butyrate is able to promote differentiation of T-regulatory cells, these results might consequently indicate that a defect of butyrate production might lead to both reduced T-reg responses and activation of immunopathological T-effector responses (36).

\section{HISTOLOGICAL CHANGES IN THE GUT OF RHEUMATOID ARTHRITIS PATIENTS}

The prevalence of morphologic bowel lesions in patients with rheumatoid arthritis (RA) was studied by Porzio et al. (37) in 1997. During the endoscopic examination, gut inflammation was observed in $11 \%$ of RA patients. At the histological examination $15 \%$ of RA patients showed ultrastructural alterations and at the electron microscopic 50\% displayed significant al- 
terations (37). According to the presence of subclinical gut inflammation, an increased intestinal permeability was found in RA patients, even though essentially linked to the intake of anti-inflammatory drugs (38). Nissinen et al. studied whether inflammation in the RA gut is associated with immune system activation (39). In particular these Authors examined the expression of chemokine receptors and cytokine-specific mRNA in intestinal biopsy samples from patients with RA (39). The Authors demonstrated that the mRNA expression of CCR4, CCR5 and IL10, but not of IL2, IFNgamma, TNFalpha, or TGFbeta, in intestinal biopsy samples was increased in patients with RA in comparison with control subjects (39). Specific alterations in the gut and oral microbiomes in individuals with RA has been recently demonstrated, suggesting potential ways of using microbiome composition for prognosis and diagnosis (40). In particular, alterations in the gut, dental or saliva microbiome distinguished individuals with RA from healthy controls, were correlated with clinical measures and could be used to stratify individuals on the basis of their response to therapy. In particular, Haemophilus spp. were depleted in individuals with RA gut and negatively correlated with levels of serum autoantibodies, whereas Lactobacillus salivarius was over-represented in the gut of individuals with RA and was present in increased amounts in cases of very active RA (40). Functional studies revealed that the redox environment, transport and metabolism of iron, sulphur, zinc and arginine were altered in the microbiota of individuals with RA. Supporting the role of RA intestinal dysbiosis in the pathogenesis of the disease is the recent demonstration that probiotic supplementation improves inflammatory status in patients with RA (41).

\section{HISTOLOGICAL CHANGES IN THE GUT OF SYSTEMIC LUPUS ERYTHEMATOSUS PATIENTS}

Systemic lupus erythematosus (SLE) is an autoimmune inflammatory disease char- acterized by the presence of a wide range of autoantibodies potentially affecting every system and organ (42). Although gastrointestinal manifestations, sometimes life-threatening, are common in SLE patients, it is thought that most of them are iatrogenic or due to infection. However, since SLE gastrointestinal manifestations are indistinct and may not have abdominal symptoms, it is possible that their incidence might be underestimated. Takeno et al. demonstrated that $60 \%-70 \%$ of SLE patients had autoptic evidence of peritonitis, whereas only around $10 \%$ of them were recognized clinically (43).

The most frequent gastrointestinal manifestation in SLE patients is represented by lupus mesenteric vasculitis (LMV), which is one of the main causes of acute abdominal pain in SLE patients. It can be classified as an acute ischemic enteritis that involves mainly small intestine and chronic multiple ulcers and occurs mainly in the colon. The proposed pathogenic mechanisms of LMV are the occurrence of inflammatory vasculitis secondary to immune complex deposition and thrombosis of the intestinal vessels secondary to circulating antiphospholipid antibodies (43).

Protein-losing gastroenteropathy (PLGE), characterized by profound edema and severe hypoalbuminemia secondary to excessive loss of serum protein from the gastrointestinal tract, is sometimes observed in SLE patients (44). In many patients, the typical features of PLGE develop before the diagnosis of SLE. In Zheng's study, $53.3 \%$ of patients had PLGE as the initial presentation of SLE, but PLGE could also occur 17 years after the establishment of SLE (44).

The pathogenic mechanisms of SLE-associated PLGE are still unclear. Different histological alterations such as mucosal ulceration, non-necrotizing mesenteric or intestinal vessel vasculitis, increase in capillary permeability caused by complement activation, pro-inflammatory cytokines, and intestinal lymphangiectasia have been described in SLE-associated PLGE (44). Less frequently, SLE patients might develop intestinal pseudo-obstruction (IPO), 
which is the result of the alteration of the visceral smooth muscle, the enteric nerve and of the visceral automatic nervous system. Intestinal leiomyocyte damage due to vasculitis leading to chronic ischemia of the bowel smooth muscle has been proposed as the main pathogenic alterations responsible for SLE-associated IPO (45, 46). Other rare gastrointestinal manifestations in SLE patients are represented by the co-existence of celiac disease (47), eosinophilic gastroenteritis and inflammatory bowel disease $(48,49)$.

\section{HISTOLOGICAL CHANGES IN THE GUT OF SYSTEMIC SCLEROSIS PATIENTS}

Gastrointestinal involvement is present in nearly all patients with SSc and often represents the first non-Raynaud phenomenon symptom (50). Gastrointestinal manifestations of mild or severe entity may occur in patients with both limited or diffuse disease. The entire gastrointestinal tract may be involved as the consequence of smooth muscle atrophy and fibrosis within the gastrointestinal mucosa and of vascular intimal proliferation (50). The most frequent histological alterations are represented by mucosal telangiectasias, pseudo-obstruction and large-intestinal mucosal muscular atrophy (51).

\section{CONCLUSIONS}

Intestinal histological alterations are common in rheumatic diseases. In many rheumatic diseases intestinal inflammation appears to be linked to dysbiosis and possibly represents the common denominator in the pathogenesis of different rheumatic diseases. The exact contribution of genetic factors in the development of intestinal inflammation in rheumatic diseases needs to be clarified. Further studies are strongly required to clarify the exact contribution of the gut in the pathogenesis of the individual rheumatic diseases.

Disclosures: the authors declare no conflict of interests.

\section{REFERENCES}

1. Peterson LW, Artis D. Intestinal epithelial cells: regulators of barrier function and immune homeostasis. Nature Rev Immunol. 2014; 14: 141-53.

2. Ley RE, Hamady M, Lozupone C, et al. Evolution of mammals and their gut microbes. Science. 2008; 320: 1647-51.

3. Costello ME, Ciccia F, Willner D, et al. Brief report: intestinal dysbiosis in ankylosing spondylitis. Arthrit Rheum. 2015; 67: 686-91.

4. Girbovan A, Sur G, Samasca G, Lupan I. Dysbiosis a risk factor for celiac disease. Med Microbiol Immunol. 2017; 206: 83-91.

5. Lippert K, Kedenko L, Antonielli L, et al. Gut microbiota dysbiosis associated with glucose metabolism disorders and the metabolic syndrome in older adults. Benef Microb. 2017; 8: 545-56.

6. Maeda Y, Takeda K. Role of gut microbiota in rheumatoid arthritis. J Clin Med. 2017; 6: 6.

7. Gaston JSH. Recent advances in understanding spondyloarthritis. F1000 Res. 2017; 6: 304.

8. Bazzocchi A, Aparisi Gomez MP, Guglielmi G. Conventional radiology in spondyloarthritis. Radiol Clin North Am. 2017; 55: 943-66.

9. Garg N, van den Bosch F, Deodhar A. The concept of spondyloarthritis: where are we now? Best Pract Res Clin Rheumatol. 2014; 28: 663-72.

10. Wendling D. The gut in spondyloarthritis. Joint Bone Spine Rev Rheum. 2016; 83: 401-5.

11. Mielants H, Veys EM, De Vos M, et al. The evolution of spondyloarthropathies in relation to gut histology. I. Clinical aspects. J Rheumatol. 1995 ; 22: 2266-72.

12. Mielants H, Veys EM, Cuvelier C, et al. The evolution of spondyloarthropathies in relation to gut histology. II. Histological aspects. J Rheumatol. 1995; 22: 2273-8.

13. Mielants H, Veys EM, Cuvelier C, et al. The evolution of spondyloarthropathies in relation to gut histology. III. Relation between gut and joint. J Rheumatol. 1995; 22: 2279-84.

14. Mielants H, Veys EM, Cuvelier C, de Vos $\mathrm{M}$. Ileocolonoscopic findings in seronegative spondylarthropathies. Br J Rheumatol. 1988; 27: 95-105.

15. Ciccia F, Bombardieri M, Principato A, et al. Overexpression of interleukin-23, but not interleukin-17, as an immunologic signature of subclinical intestinal inflammation in ankylosing spondylitis. Arthrit Rheum. 2009; 60: 955-65.

16. Ciccia F, Accardo-Palumbo A, Alessandro R, et al. Interleukin-22 and interleukin-22-producing NKp44+ natural killer cells in subclinical gut inflammation in ankylosing spondylitis. Arthrit Rheum. 2012; 64: 1869-78.

17. Ciccia F, Bombardieri M, Rizzo A, et al. Overexpression of paneth cell-derived anti-micro- 
bial peptides in the gut of patients with ankylosing spondylitis and subclinical intestinal inflammation. Rheumatol. 2010; 49: 2076-83.

18. Ciccia F, Guggino G, Rizzo A, et al. Dysbiosis and zonulin upregulation alter gut epithelial and vascular barriers in patients with ankylosing spondylitis. Ann Rheum Dis. 2017; 76: 1123-32.

19. Spadoni I, Zagato E, Bertocchi A, et al. A gutvascular barrier controls the systemic dissemination of bacteria. Science. 2015; 350: 830-4.

20. El Asmar R, Panigrahi P, Bamford P, et al. Host-dependent zonulin secretion causes the impairment of the small intestine barrier function after bacterial exposure. Gastroenterol. 2002; 123: 1607-15.

21. Klingberg E, Strid H, Stahl A, et al. A longitudinal study of fecal calprotectin and the development of inflammatory bowel disease in ankylosing spondylitis. Arthrit Res Ther. 2017; 19: 21.

22. Schatteman L, Mielants H, Veys EM, et al. Gut inflammation in psoriatic arthritis: a prospective ileocolonoscopic study. J Rheum. 1995; 22: 680-3.

23. Ciccia F, Rizzo A, Triolo G. Subclinical gut inflammation in ankylosing spondylitis. Curr Opin Rheumatol. 2016; 28: 89-96.

24. Scarpa R, Manguso F, D’Arienzo A, et al. Microscopic inflammatory changes in colon of patients with both active psoriasis and psoriatic arthritis without bowel symptoms. J Rheumatol. 2000; 27: 1241-6.

25. Ciccia F, Guggino G, Ferrante A, et al. Interleukin-9 overexpression and Th9 polarization characterize the inflamed gut, the synovial tissue, and the peripheral blood of patients with psoriatic arthritis. Arthrit Rheumatol. 2016; 68: 1922-31.

26. Scher JU, Littman DR, Abramson SB. Microbiome in inflammatory arthritis and human rheumatic diseases. Arthrit Rheumatol. 2016; 68: 35-45.

27. Mielants H, Veys EM, Joos R, et al. Late onset pauciarticular juvenile chronic arthritis: relation to gut inflammation. J Rheumatol. 1987; 14: 459-65.

28. Mielants H, Veys EM, Cuvelier C, et al. Gut inflammation in children with late onset pauciarticular juvenile chronic arthritis and evolution to adult spondyloarthropathy - a prospective study. J Rheumatol. 1993; 20: 1567-72.

29. Pichler J, Ong C, Shah N, et al. Histopathological features of gastrointestinal mucosal biopsies in children with juvenile idiopathic arthritis. Pediatr Res. 2016; 79: 895-901.

30. Di Paola M, Cavalieri D, Albanese D, et al. Alteration of fecal microbiota profiles in juvenile idiopathic arthritis. Associations with HLAB27 allele and disease status. Front Microbiol. 2016; 7: 1703.

31. Aggarwal A, Sarangi AN, Gaur P, et al. Gut microbiome in children with enthesitis-related arthritis in a developing country and the effect of probiotic administration. Clin Exp Immunol. 2017; 187: 480-9.

32. Valenti S, Gallizzi R, De Vivo D, Romano C. Intestinal Behçet and Crohn's disease: two sides of the same coin. Pediatr Rheum Online J. 2017; 15: 33.

33. Asakura H, Morita A, Morishita T, et al. Histopathological and electron microscopic studies of lymphangiectasia of the small intestine in Behçet's disease. Gut. 1973; 14: 196-203.

34. Ferrante A, Ciccia F, Principato A, et al. A Th1 but not a Th17 response is present in the gastrointestinal involvement of Behçet's disease. Clin Exp Rheum. 2010; 28: S27-30.

35. Shimizu J, Kubota T, Takada E, et al. Bifidobacteria abundance-featured gut microbiota compositional change in patients with Behçet's disease. PLoS One. 2016; 11: e0153746.

36. Consolandi C, Turroni S, Emmi G, et al. Behçet's syndrome patients exhibit specific microbiome signature. Autoimmun Rev. 2015; 14: 269-76.

37. Porzio V, Biasi G, Corrado A, et al. Intestinal histological and ultrastructural inflammatory changes in spondyloarthropathy and rheumatoid arthritis. Scand J Rheumatol. 1997; 26: 92-8.

38. Mielants H, Goemaere S, De Vos M, et al. Intestinal mucosal permeability in inflammatory rheumatic diseases. I. Role of antiinflammatory drugs. J Rheum. 1991; 18: 389-93.

39. Nissinen R, Leirisalo-Repo M, Nieminen AM, et al. Immune activation in the small intestine in patients with rheumatoid arthritis. Ann Rheum Dis. 2004; 63: 1327-30.

40. Zhang X, Zhang D, Jia H, et al. The oral and gut microbiomes are perturbed in rheumatoid arthritis and partly normalized after treatment. Nature Med. 2015; 21: 895-905.

41. Vaghef-Mehrabany E, Alipour B, HomayouniRad A, et al. Probiotic supplementation improves inflammatory status in patients with rheumatoid arthritis. Nutrition. 2014; 30: 430-5.

42. Gualtierotti R, Biggioggero M, Penatti AE, Meroni PL. Updating on the pathogenesis of systemic lupus erythematosus. Autoimmun Rev. 2010; 10: 3-7.

43. Takeno M, Ishigatsubo Y. Intestinal manifestations in systemic lupus erythematosus. Intern Med. 2006; 45: 41-2.

44. Tian XP, Zhang X. Gastrointestinal involvement in systemic lupus erythematosus: insight into pathogenesis, diagnosis and treatment. World J Gastroenterol. 2010; 16: 2971-7.

45. Pardos-Gea J, Ordi-Ros J, Selva A, et al. Chronic intestinal pseudo-obstruction associated with biliary tract dilatation in a patient with systemic lupus erythematosus. Lupus. 2005; 14: 328-30.

46. Ceccato F, Salas A, Gongora V, et al. Chronic intestinal pseudo-obstruction in patients with 
systemic lupus erythematosus: report of four cases. Clin Rheumatol. 2008; 27: 399-402.

47. Gupta D, Mirza N. Systemic lupus erythematosus, celiac disease and antiphospholipid antibody syndrome: a rare association. Rheum Int. 2008; 28: 1179-80.

48. Medeiros DA, Isenberg DA. Systemic lupus erythematosus and ulcerative colitis. Lupus. 2009; 18: 762-3.

49. Su KY, Tsai ST, Tsay SH, et al. A patient with systemic lupus erythematosus and Crohn's disease complicated with massive lower gastrointestinal bleeding, mimicking intestinal vasculitis. Lupus. 2008; 17: 1049-50.

50. Kumar S, Singh J, Rattan S, et al. Review article: pathogenesis and clinical manifestations of gastrointestinal involvement in systemic sclerosis. Aliment Pharmacol Ther. 2017; 45: 883-98.

51. Butt S, Emmanuel A. Systemic sclerosis and the gut. Expert Rev Gastroenterol Hepatol. 2013; 7: 331-9. 